\title{
Spatiotemporal Data Mining with Cellular Automata
}

\author{
Karl Fu and Yang Cai \\ Carnegie Mellon University, Visual Intelligence Studios, \\ Cylab, CIC-2218, 4720 Forbes Ave. \\ Pittsburgh, PA 15213 USA \\ ycai@cmu.edu
}

\begin{abstract}
In this paper, we describe a cellular automata model for predicting biological spatiotemporal dynamics in an imagery data flow. The Bayesian probability-based algorithm is used to estimate the algal formation in a twodimensional space. The dynamics of the cellular artificial life is described with diffusion, transport, collision and deformation. We tested the model with the historical data, including parameters, such as time, position and temperature.
\end{abstract}

\section{Introduction}

Spatiotemporal data are widely used in remote sensing community. The imagery data usually contain multi-spectrum information such as visible, infrared, UV, or microwave signals. Scientists need to find out the physical properties behind the data flow and predict the future trends such as global warming, flood, harmful algae blooms or river plumes. However, in many cases, the inverse physics process is complicated. Heuristics has to be used for approximate estimations.

Cellular Automata (CA) has been used for modeling artificial lives [2-13], as well as, at the large scale, urban and economic dynamics [5]. Furthermore, CA has been employed in simulating and verifying various types of physical interactions such as the expansion patterns of different lattice gases, surface tension of liquids and diffusion-limited aggregation [13]. Although CA's rules are simple and local, it can simulate complex spatiotemporal interactions that are challenging to other computational methods. In this paper, we present a two-dimensional cellular artificial life model for predicting the spatiotemporal dynamics in an imagery flow. The model consists of a set of parameters and rules that control spatiotemporal dynamics in form of a shape, such as diffusion, transport, collision. Similar to the conventional cellular automata, artificial lives in this model interact with their neighbors individually. However, they form a coherent shape as a lump.

\section{Formation of the Artificial Life}

Given historical spatiotemporal data of the location of the alga, it is possible to model the formation of the artificial life in a cellular grid. In this model, we consider the location of the algae, time and physical parameters, such as temperature and wind. 
For historical images $I_{j}$ where $j \in\{1,2,3, \ldots, i\}, I_{j}(x, y)$ is a logical ' 1 ' if the pixel is occupied by algae, and a logical ' 0 ' if it's not occupied by an alga where $x \in\{1,2,3, \ldots, a\}, y \in\{1,2,3, \ldots, b\}$. The total number of images given is $i$, the horizontal dimensions of the image is denoted $a$, and the vertical dimensions of the image is denoted $b$. To predict the location of the algal, evidence such as the time of the year, or the surface temperature are given as condition. Let $e$ be the condition given (i.e. temperature and/or time) and $I_{j}^{e}$ be the predicted image given $e$ as condition:

$$
\begin{gathered}
P\left(I_{j}(x, y) \mid e\right)=P\left(e \mid I_{j}(x, y)\right) * P\left(I_{j}(x, y)\right) / P(e) \\
I_{j}^{e}(x, y)=\left(P\left(I_{j}(x, y) \mid e\right) \geq \alpha,\right.
\end{gathered}
$$

where $\alpha$ is a threshold for when $I_{j}^{e}(x, y)$ is inhabited by algae.

The probabilities under those conditions/evidences, such as time and temperature, can be calculated. The Figure 1 shows examples of the estimated target locations.

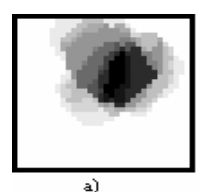

a)

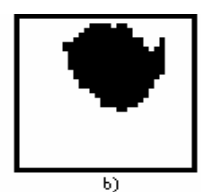

b)

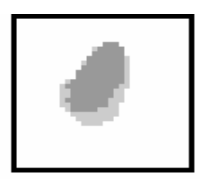

c)

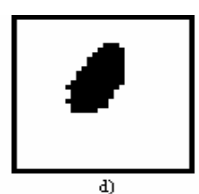

d)

Fig. 1. a) Probability of location being inhabited by algae using a single input temperature $=15^{\circ} \mathrm{C}$. b) predicted image of using $15^{\circ} \mathrm{C}$ as input $\alpha=.3$, c) probability of location being inhabited by algae using a single input time = January, d) predicted image of using January as input.

Prediction using one input becomes much more inaccurate during months with cold temperature because during certain time of the year no alga is detected when the temperature falls below $15^{\circ} \mathrm{C}$. In Figure 1 , a) and c) are poor probability models because of lack of evidences for the Bayesian model to make an accurate prediction; this is indicated by the large number of cells in each image with very low probability (light colored cells).

\section{Dynamics of the Artificial Life}

The growth diffusion function changes the object shape by a predefined structuring element. The dilation or erosion morphology is based on the assumption of a glasslike surface where no friction exists. They can only deform the outline of a shape uniformly based on the population. It doesn't reflect the intrinsic or external nonlinear or random factors that contribute to the shape process. To simulate the nonuniform shape growth, we investigate the percolation clustering [18-19]. Figure 2 shows an example of the percolation process started from a given shape.

Algae's growths and movements are often affected by environmental factors such as external forces: winds and currents, and the presence of obstacles. A collision causes deformation. The level of a deformation depends on the elasticity of an object. For a 


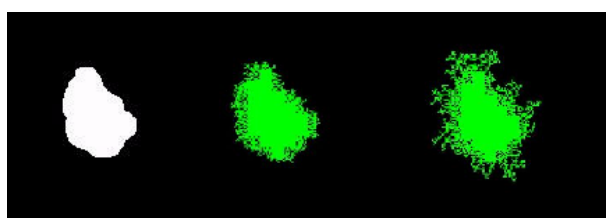

Fig. 2. Diffusion with resistance

rigid object, it will stop at the collision contact point and keep the same shape. We classify objects as rigid, elastic and flexible. Rigid objects don't deform after the impact. Flexible objects, on the other hand, completely deform along the outline of the collided hard objects. The two extreme cases are rather easy to describe. Assume we have an elastic object and a rigid object, we use the following rules to model the deformation: 1) Detect the collision points; 2) Move a portion of the collided cells to 'sideways', the ratio is proportion to the elasticity of the object. The bigger elasticity, the more cells move sideways. 3) Continue to deform until the motion stops.

\section{Results}

We verify the algorithm with a case study of predicting the movement of harmful algal bloom (HAB) of Karena Brevis. In this case, the HABs are 'lumps', rather than individual cells. The input data are simulated satellite images and cell counts. The predicted results from the model are compared to the actual images under the same conditions, such as time and temperature. The error of the 30 trials is within $5.40 \%$. Without using both variables, the error is $8.24 \%$. While using both variables, the error is only $4.68 \%$. With increasing amount of evidences, the prediction accuracy increases substantially.

\section{Conclusions}

In this paper, we use the cellular artificial life to anticipate complex spatiotemporal phenomena of algal blooms, including formation, diffusion, collision, transport, and deformation. By combining the statistical model with cellular automata, we found the new approach yields decent accuracy to predict the algal location and the shape dynamics. However, the predictability of the hybrid model depends on the historical data and its fitness to its complex environment.

\section{Acknowledgement}

This study is supported by NASA ESTO grant AIST-QRS-04-3031. We are indebted to our collaborators in NOAA, Dr. Richard Stumpf and Mr. Timothy Wynne for their expertise in oceanography and databases. The authors appreciate the comments and suggestions from Dr. Karen Meo, Dr. Kai-Dee Chu, Dr. Steven Smith, Dr. Gene Carl Feldman and Dr. James Acker from NASA. Also, many thanks to Professor Christos Faloulus, graduate student Daniel Chung of Carnegie Mellon University for their input. 


\section{References}

[1] Lewin, K. Field Theory in Social Science. Harper and Row, 1951

[2] von Neumann, John, 1966, The Theory of Self-reproducing Automata, A. Burks, ed., Univ. of Illinois Press, Urbana, IL.

[3] Gardtner, M. 1970. Mathematical game. The fantastic combinations of John Conroy's new solitaire game "life". Scientific American vol. 223, pp. 120-120.

[4] Margolus, N. and Toffoli, T. Cellular Automata Machines: A New Environment for Modeling. The MIT Press, 1987.

[5] Yeh, A. G. O. and Li, X. Urban Simulation Using Neural Networks and Cellular Automata for Land Use Planning. Symposium on Geospatial Theory, Processing and Applications, Ottawa 2002.

[6] Wolfram, S. Statistical Mechanics of Cellular Automata. Rev. Mod. Phys. 55, 601-644, 1983.

[7] Wolfram, S. Universality and Complexity in Cellular Automata. Physica D 10, 1-35, 1984.

[8] Wolfram, S. Twenty Problems in the Theory of Cellular Automata. Physica Scripta T9, 170-183, 1985.

[9] Wolfram, S. A New Kind of Science. Champaign, IL: Wolfram Media, pp. 60-70 and 886, 2002.

[10] Wolfram, S. Cellular automata as models for complexity, Nature 311:419 (1984).

[11] Wolfram, S. Statistical mechanics of cellular automata, Rev. Mod. Phys. 55:601 (1983).

[12] Vichniac, G. Simulating physics with cellular automata, Physica 10D:96 (1984).

[13] Hardy, J., O. de Pazzis, and Y. Pomeau, Molecular dynamics of a classical lattice gas: transport properties and time correlation functions, Phys. Rev. A13:1949 (1976).

[14] Leyton, M. Symmetry, Causality, Mind, MIT Press, 1999

[15] Sonika, M. etc. Image Processing, Analysis, and Machine Vision, PWS Publishing, 1999

[16] Zhou, S., Chellappa, R. and Moghaddam, B. Visual tracking and recognition using apprearance-adaptive models in particle filters. IEEE Trans. On Image Processing, Vol. 13, No. 11, pp. 1491-1506, 2004

[17] Bandini, S. and Pavesi, G. Controlled generation of two-dimensional patterns based on stochastic cellular automata, Future Generation Computer Systems, vol. 18 (2002) 973 981

[18] Stauffer, D., Aharony, A. Introduction to percolation Theory, Taylor \& Francis, London, 1992

[19] Essam, J.W. Percolation theory, Rep. Prog. Phys. 43:833 (1980) 